\title{
STUDY OF SILICON PHOTOMULTIPLIERS FOR THE GRIPS CALORIMETER MODULE
}

\author{
Alexei Ulyanov*, Lorraine Hanlon, Sheila McBreen, Suzanne Foley \\ School of Physics, University College Dublin, Ireland \\ * corresponding author: alexey.uliyanov@ucd.ie
}

\begin{abstract}
GRIPS is a proposed gamma-ray (200 keV to $80 \mathrm{MeV}$ ) astronomy mission, which incorporates a pair-creation and Compton scattering telescope, along with X-ray and infrared telescopes. It will carry out a sensitive all-sky scanning survey, investigating phenomena such as gamma-ray bursts, blazars and core collapse supernovae. The main telescope is composed of a Si strip detector surrounded by a calorimeter with a fast scintillator material. We present the initial results of a study which considers the potential use of silicon photomultipliers in conjunction with the scintillator in the GRIPS calorimeter module.
\end{abstract}

KEYWORDS: silicon photomultiplier; GRIPS; gamma-ray; future.

\section{INTRODUCTION}

GRIPS (Gamma-Ray Imaging, Polarimetry and Spectroscopy) [1, 2] is a proposed $\gamma$-ray astronomy mission, which will perform a sensitive all-sky scanning survey from $200 \mathrm{keV}$ to $80 \mathrm{MeV}$. With respect to previous missions (e.g. INTEGRAL, COMPTEL, EGRET) the sensitivity in this energy range will be improved by at least an order of magnitude. The GRIPS mission will investigate $\gamma$-ray bursts and blazars, the mechanisms behind supernova explosions, nucleosynthesis and spallation, the origin of positrons in our Galaxy, and the nature of radiation processes and particle acceleration in extreme cosmic sources including pulsars and magnetars.

As its primary instrument, GRIPS will have a combined Compton scattering and pair creation telescope called the Gamma-Ray Monitor (GRM). In addition, a separate satellite will carry two auxiliary telescopes: an X-Ray Monitor (XRM) and an InfraRed Telescope (IRT), which will help to accurately localise GRB counterparts and measure their redshifts. Similar to previous Compton and pair creation telescopes, the GRM design envisages two separate detectors: a silicon tracker, in which the initial Compton scattering or pair conversion takes place, and a calorimeter, which absorbs and measures the energy of the secondaries. The calorimeter employs $\mathrm{LaBr}_{3}$ scintillator crystals to achieve an improved energy resolution (about $3 \%$ at $1 \mathrm{MeV}$ ) and faster response times compared to traditional scintillator materials such as NaI, CsI, or BGO.

For optimum angular resolution GRM requires a finely segmented calorimeter with $\sim 10^{5}$ readout channels. This precludes the use of bulky, fragile, highvoltage photomultiplier tubes, traditionally employed for the readout of scintillator crystals. PIN diodes are not suitable because they have no internal gain and produce signals with a low signal-to-noise ratio,

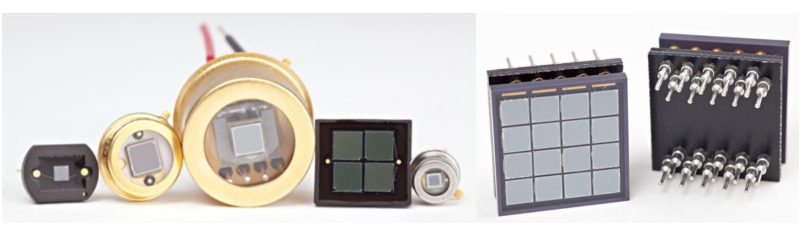

FigURE 1. A selection of the silicon photomultipliers produced by SensL.

resulting in poor energy resolution. The GRM relies on development of advanced semiconductor photodetectors such as silicon drift detectors (SDD). Recent technological advances in the development of silicon photomultipliers (SiPM) make them a promising option for the GRM calorimeter readout. These detectors combine the high gain of traditional photomultipliers with low-voltage operation, robustness, low mass and compact design typical for semiconductor devices.

The purpose of this study is to evaluate the possibility of using novel SiPMs for the calorimeter readout in the GRIPS mission and to optimise the design of a calorimeter module. This work is a collaboration between the Space Science Group in University College Dublin and SensL Technologies Ltd (http://www.sensl.com), an Irish company that has developed the novel SiPMs. Another Irish company, ACRA Control Ltd., is involved in the design of a suitable Payload Interface Unit for the instrument. We present here the results of an initial study, which has focused on the technical requirements of the GRM calorimeter.

\section{SiPM DEVELOPMENTS BY SENSL}

SensL have designed SiPMs to meet the needs of a variety of applications from analytical instruments, hazard and threat detection to nuclear medicine and 

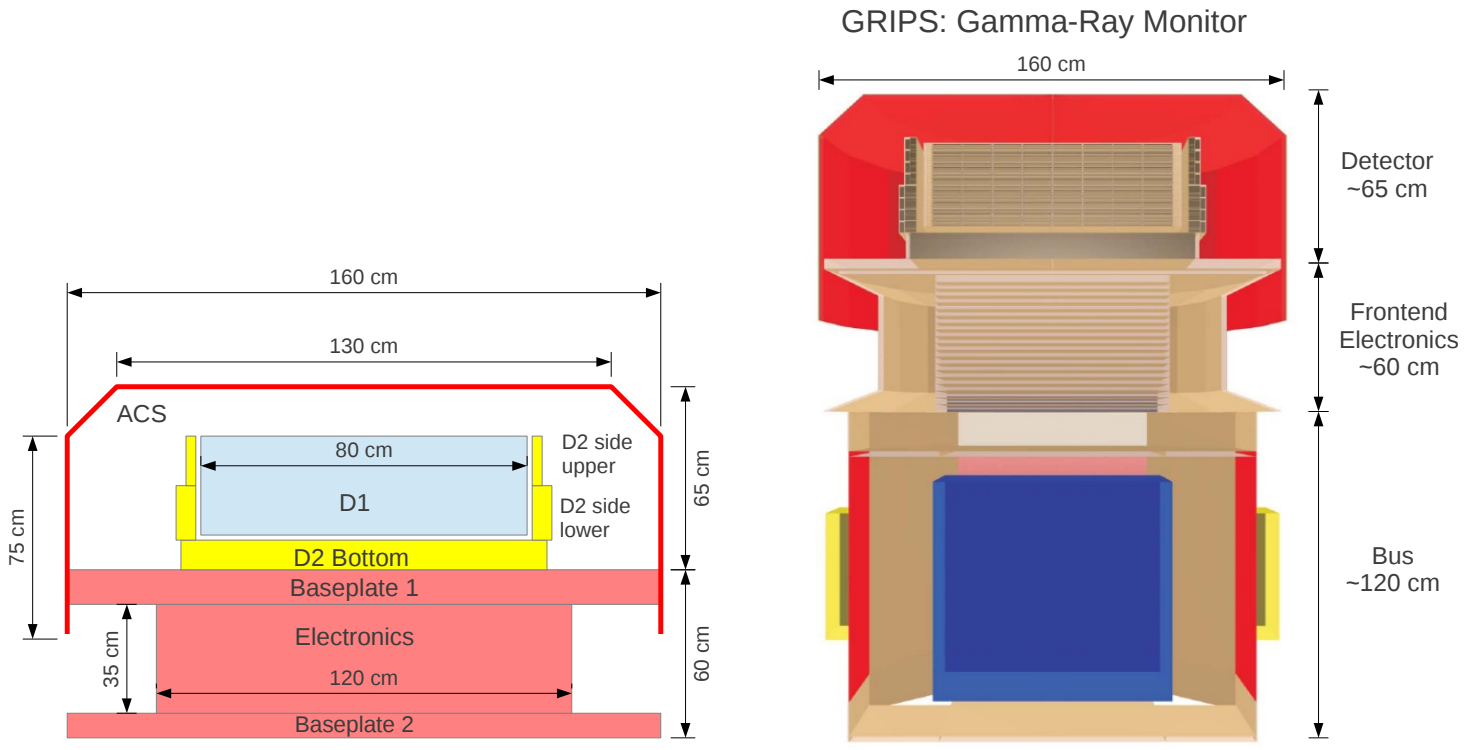

Figure 2. Left: Geometry and size of the GRM detector used in the simulations. Right: Expected size and configuration of GRM and related electronics on a generic satellite bus.

process monitoring. The current product range includes sensors of three sizes ( $1 \mathrm{~mm}, 3 \mathrm{~mm}$ and $6 \mathrm{~mm}$ ) available in various packages (Fig. 1), with 19096 microcells per $6 \mathrm{~mm}$ detector. In addition, a $4 \times 4$ array of SiPM pixels is available. The array permits close packing on all four sides allowing for a detection area that can be as large or small as required by the specific application. It is the first commercially available detector of its kind. Larger $8 \times 8 \mathrm{SiPM}$ custom arrays have also been produced.

\section{GRM MODEL AND SIMULATION ENVIRONMENT}

We have carried out Monte-Carlo simulations to understand how the properties of different detector components influence the performance of the whole GRM detector. For the simulations we used the MEGAlib software package [3, 4]. The package includes tools for detector description and simulation of particle interactions with matter using the Geant4 toolkit [5]. It also provides dedicated tools for event reconstruction and high-level analysis for Compton and pair telescopes.

The simulations performed in the scope of this project used the GRM baseline model from [2 including the GRM instrument, the support tube with the electronics box, but not the spacecraft (Fig. 2). The tracker consists of 64 layers each containing a mosaic of $8 \times 8$ double-sided Si strip detectors of area $10 \times 10 \mathrm{~cm}^{2}$, each of those detectors having 96 strips per side. The layers are spaced at a distance of $5 \mathrm{~mm}$. The calorimeter is made of $\mathrm{LaBr}_{3}$ prisms with $5 \times 5 \mathrm{~mm}^{2}$ cross-section. The upper half of the calorimeter side walls feature scintillators of $2 \mathrm{~cm}$ length and the lower half has $4 \mathrm{~cm}$ thick walls. The side wall crystals are read out by one photodetector each. The bottom calorimeter is $8 \mathrm{~cm}$ thick and is read out on both ends of the crystals to achieve a depth resolution of the energy deposits. The whole detector is surrounded by a plastic scintillator counter that acts as an anticoincidence shield against charged particles.

The background for the GRIPS mission (equatorial low-earth orbit) is dominated by diffuse cosmic photons and albedo photons coming from the Earth's atmosphere. These were the only background components included in the simulations.

To carry out CPU intensive simulations we use the Pascal IBM HPC Cluster at UCD, which is a 720 core supercomputer running the Red Hat Enterprise Linux 5.2 operating system. The cluster has 28 nodes allocated to the School of Physics. Each node has $16 \mathrm{~GB}$ of RAM and two Xeon $3 \mathrm{GHz}$ processors yielding eight cores per node.

\section{INITIAL RESULTS}

The calorimeter energy resolution is simulated in MEGAlib by adding Gaussian noise to ideal energy deposits in sensitive volumes produced by particles in the Geant simulations. The influence of the calorimeter energy resolution on the overall GRM sensitivity was investigated by multiplying the baseline energy resolution (a function of energy) by a scale factor. The resulting effect on the GRM sensitivity is shown in Figs. 3 and 4 for a variety of sources. While the calorimeter resolution has a strong impact on the narrow line source sensitivity, it has only a moderate influence on the continuum source sensitivity, mostly 

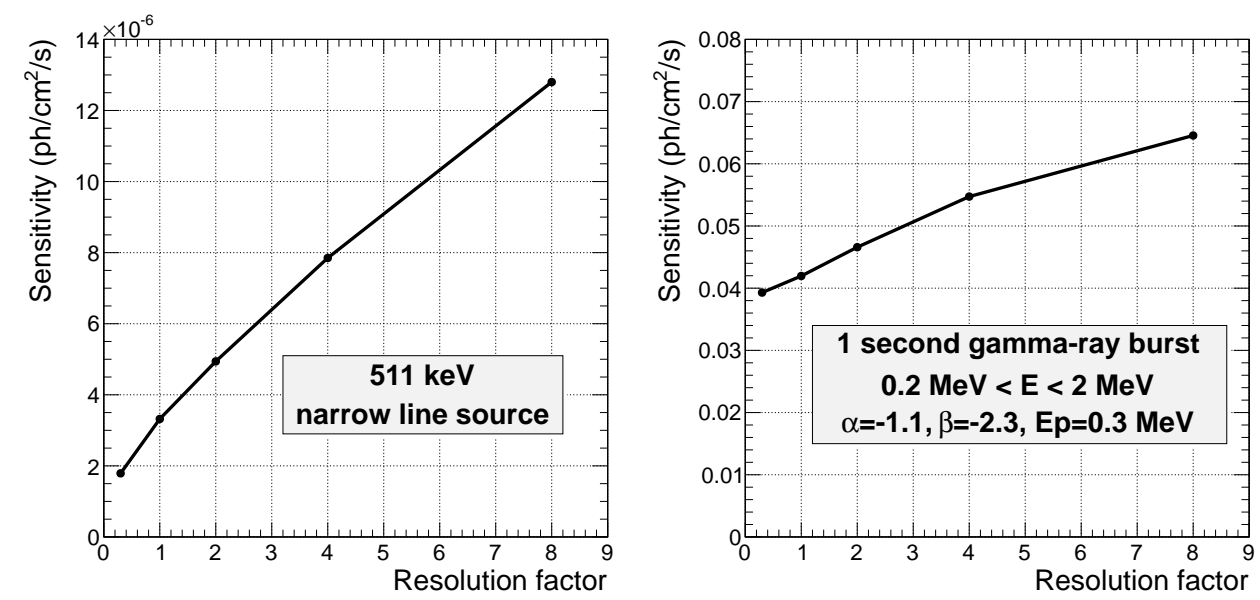

FIGURE 3. On-axis point source sensitivity versus calorimeter energy resolution (in units of the baseline resolution) for a narrow line $\left(10^{6} \mathrm{~s}\right.$ exposure) source and a continuum GRB spectrum ( $1 \mathrm{~s}$ exposure). The scale factor of 1 corresponds to the baseline energy resolution $(4.6 \%$ at $662 \mathrm{keV})$.
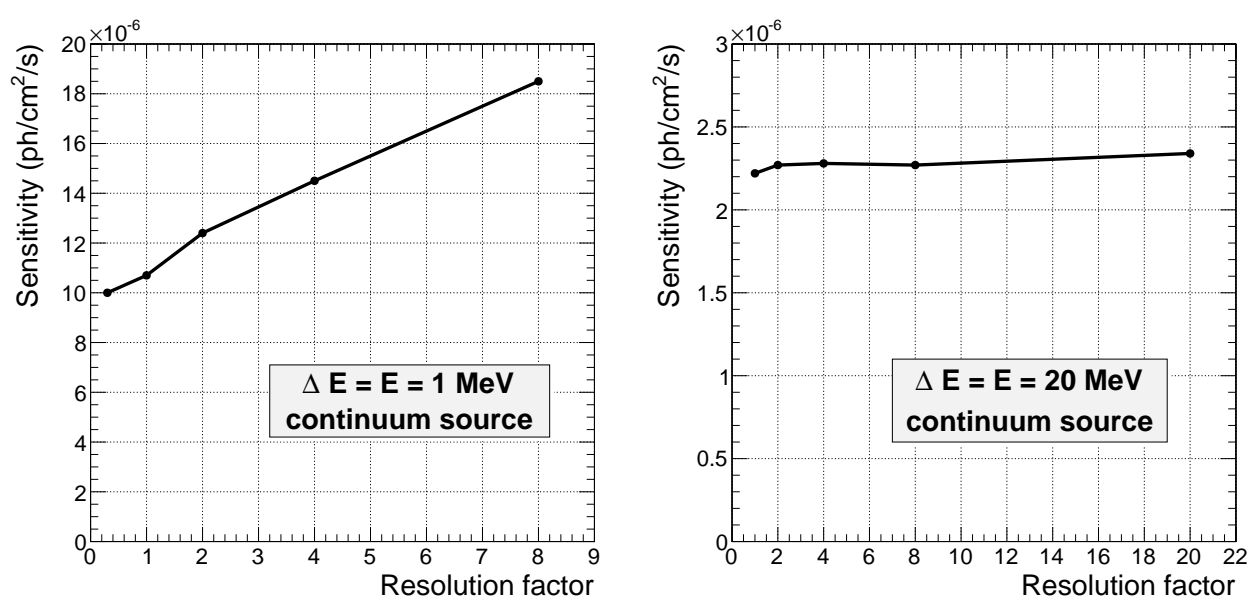

FIGURE 4. On-axis continuum point source sensitivity in a $10^{6} \mathrm{~s}$ exposure for a source with an $E^{-2}$ spectrum.
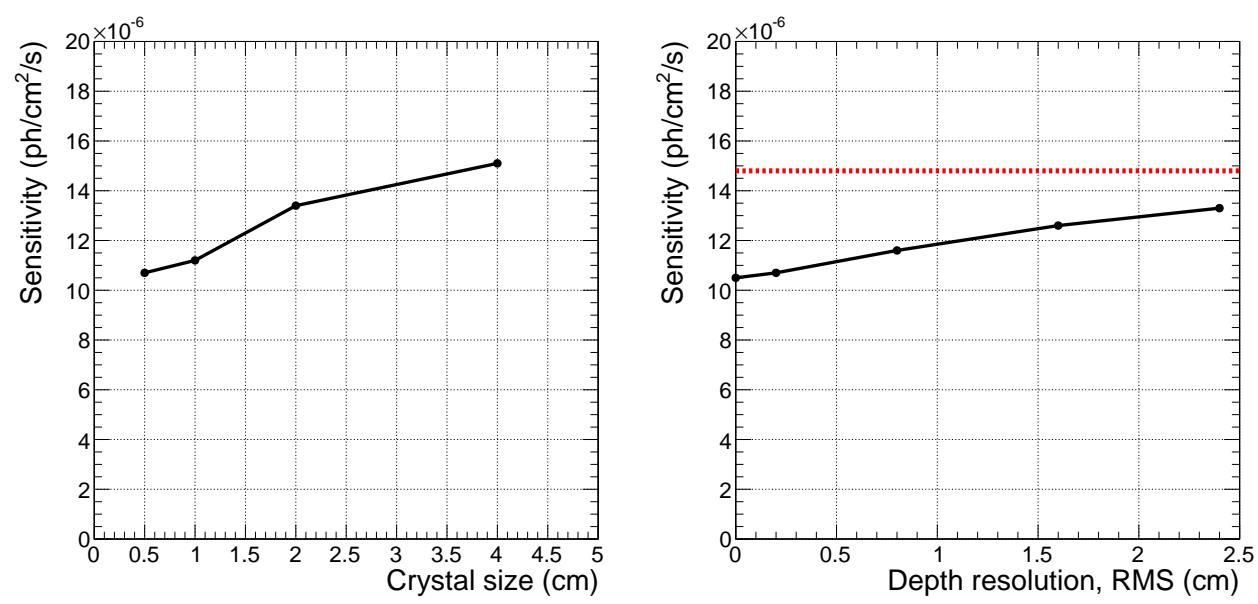

FigURE 5. On-axis continuum point source sensitivity for a $10^{6} \mathrm{~s}$ exposure versus transverse crystal size (left) and versus calorimeter depth resolution (right); $E^{-2}$ spectrum, $0.5 \mathrm{MeV}<E<1.5 \mathrm{MeV}$. The sensitivity obtained without any depth resolution is shown by the red dashed line. 


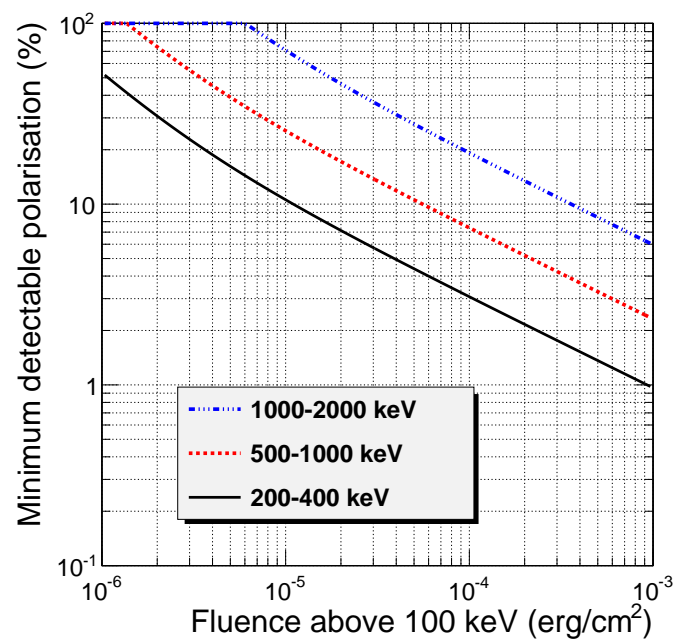

FIgURE 6. On-axis GRM polarisation sensitivity in different energy bands as a function of the GRB fluence. The GRB spectrum is modelled with the Band function $\left(\alpha=-1.1, \beta=-2.3, E_{\mathrm{p}}=0.3 \mathrm{MeV}\right)$. The polarisation sensitivity also depends on the amount of background and consequently on the GRB duration, which is taken to be $20 \mathrm{~s}$ for this analysis. The brightest GRB detected by BATSE had a fluence of $7.3 \cdot 10^{-4} \mathrm{erg} / \mathrm{cm}^{2}$ above $100 \mathrm{keV}$.

through a change in the GRM angular resolution. The calorimeter energy resolution becomes relatively unimportant at energies above $\sim 10 \mathrm{MeV}$, when the pair creation dominates over the Compton scattering and the direction of the original photon is reconstructed by the tracker. In addition, incomplete energy absorption at these energies becomes more important than the intrinsic energy resolution of the calorimeter.

The angular resolution and sensitivity of the GRM telescope depend on the calorimeter spatial resolution, which is achieved through the fine segmentation: $5 \times$ $5 \mathrm{~mm}^{2}$ crystal size is envisaged in the baseline design. This level of segmentation follows the design of the MEGA prototype [6] and has not been optimised for GRM. We considered several larger crystal sizes in our simulations to understand how the calorimeter segmentation affects the GRM performance. From the left plot in Fig. 5 we conclude that a crystal size of $\sim 1 \times 1 \mathrm{~cm}^{2}$ can be used to reduce the number of readout channels without significant impact on the GRM performance.

In the baseline design, $8 \mathrm{~cm}$ long crystals in the bottom calorimeter are read out at both ends to achieve a depth resolution of energy deposits. We estimated the GRM sensitivity with different values of the depth resolution and found that the depth resolution of $\sigma=1 \mathrm{~cm}$, or better, gives a noticeable improvement in the instrument sensitivity (right plot in Fig. 5).

Linearly polarised $\gamma$-rays preferentially Compton scatter perpendicular to the incident polarisation vector, resulting in an azimuthal scatter angle distribution modulated relative to the distribution for unpolarised photons. The polarisation sensitivity of GRM estimated for a typical $\gamma$-ray burst (GRB) spectrum is shown in Fig. 6

\section{Future WORK}

Our further work will be focused on:

- laboratory tests of the SiPMs supplied by SensL;

- a realistic software model of SiPMs, including thermal noise and finite dynamic range of the sensors;

- optimisation of the calorimeter module design comprising $\mathrm{LaBr}_{3}$ crystals and a SiPM array. Two options will be considered to achieve a depth resolution in the thick bottom calorimeter: double readout of single crystals and longitudinal segmentation.

\section{ACKNOWLEDGEMENTS}

We would like to thank Andreas Zoglauer who helped us to get started with the GRIPS simulations. This project is being supported under ESA's Strategic Initiative AO/16418/10/NL/Cbi.

\section{REFERENCES}

[1] Greiner, J et al.: Gamma-ray burst investigation via polarimetry and spectroscopy (GRIPS), Exp. Astron., 23, 2009, p.91.

[2] Greiner, J. et al.: GRIPS - Gamma-Ray Imaging, Polarimetry and Spectroscopy, arXiv:1105.1265, 2011.

[3] Zoglauer, A., Andritschke, R., Schopper, F.: MEGAlib - the medium energy gamma-ray astronomy library, New Astron. Rev., 50, 7-8, 2006, p.629.

[4] Zoglauer, A, Andritschke, R, Boggs, S et al.: MEGAlib: simulation and data analysis for low-to-medium-energy gamma-ray telescopes, SPIE, 7011E, 2008, p.101.

[5] Agostinelli, S. et al.: Geant4 - a simulation toolkit. Nucl. Instr. Meth., A506, 2003, p.250.

[6] Kanbach, G. et al.: Development and calibration of the tracking Compton/Pair telescope. Nucl. Instr. Meth., A541, 2005, p.310. 